\title{
FONTES DE CONHECIMENTOS DOS TREINADORES: ESTUDO DE CASO DAS SELEÇÕES BRASILEIRAS MASCULINAS DE HANDEBOL
}

\section{FUENTES DE CONOCIMIENTO DE LOS ENTRENADORES: ESTUDIO DE CASO DE EQUIPOS DE BALONMANO BRASILEÑOS}

\section{COACHES' KNOWLEDGE SOURCES: CASE STUDY OF BRAZILIAN MEN'S HANDBALL TEAMS}

\begin{abstract}
José Carlos Mendes ${ }^{1}$, Michel Milistetd ${ }^{2}$, Sergio José Ibáñez ${ }^{3}$ e Juarez Vieira Nascimento ${ }^{2}$
\end{abstract}
\section{spock12hand@hotmail.com; michel canhoto@hotmail.com; sibanez@uex.es;}

\author{
juarez.nascimento@ufsc.br \\ ${ }^{1}$ Universidade Estadual do Oeste do Paraná, Brasil \\ 2 Universidade Federal de Santa Catarina, Brasil \\ ${ }^{3}$ Universidad de Extremadura, España
}

Envio Original: 2020-03-17 Reenviado: 2020-10-02 Aceitado: 2020-10-21

Publicado: 2020-11-05

Doi: https://doi.org/10.15517/pensarmov.v18i2.41103

\begin{abstract}
Resumo
O estudo identificou as fontes de conhecimentos mais valorizadas pelos treinadores e treinadores assistentes das seleções de handebol masculino do Brasil nas categorias juvenil (U19), júnior (U21) e adulta. A pesquisa trata-se de um estudo de caso realizado com três treinadores e três treinadores assistentes das seleções masculinas de handebol do Brasil, sendo os dados coletados por meio do questionário de Perfil de Formação do Treinador. Os dados foram analisados por meio de estatística descritiva (mediana) e inferencial (U de Mann Whitney e Kruskal Wallis) com nível de significância de $p \leq 0,05$. Os resultados indicaram que, tanto os treinadores, como os treinadores assistentes das seleções, atribuíam maior valorização das fontes de conhecimentos procedentes da "experiência profissional" e da "formação acadêmica". Além disso, observou-se que as fontes de conhecimento procedentes da "experiência profissional" foram mais valorizadas pelos integrantes das comissões técnicas
\end{abstract}


das seleções U19 e adulta, enquanto os membros da comissão técnica da seleção U21 valorizavam mais as fontes de conhecimento procedentes da "formação acadêmica". Contudo não foram encontradas diferenças significativas nos resultados entre treinadores e treinadores assistentes, como também entre as comissões técnicas das seleções U19, U21 e adulta. A atuação profissional dos treinadores e treinadores assistentes das seleções brasileiras masculinas das categorias U19, U21 e adulta do Handebol e a construção do processo de treino do modelo de jogo proposto para as seleções estruturam-se a partir das fontes de conhecimentos procedentes da sua "experiência profissional" e da "formação acadêmica".

Palavras-chave: handebol, treinador, fontes de conhecimento, Brasil.

\begin{abstract}
The aim of the current study is to identify the knowledge sources mostly valued by coaches and assistant coaches of Brazilian male handball teams belonging to the youth (U19), junior (U21) and adult categories. Case study carried out with three coaches and three assistant coaches of Brazilian male handball teams; data were collected through the Coach Training Profile Questionnaire and subjected to descriptive (median) and inferential statistics (U Mann Whitney and Kruskal Wallis tests), at significance level $p \leq 0.05$.Coaches and assistant coaches of the national handball teams have attributed greater value to knowledge sources such as "professional experience" and "academic training". In addition, "professional experience" was the knowledge source mostly valued by technical commission members of the U19 and adult categories, whereas members of the technical committee of the U21 category have mostly valued "academic training" as knowledge source. However, no significant differences were observed in results between coaches and assistant coaches, as well as among the technical commissions of the U19, U21 and adult categories. The professional performance of coaches and assistant coaches of Brazilian male handball teams belonging to the $U 19, \mathrm{U} 21$ and adult categories and the construction of the training process associated with the match model proposed for the teams are structured based on knowledge sources such as " professional experience "and" academic training ".
\end{abstract}

Keywords: handball, coach, knowledge sources, Brazil. 


\section{INTRODUÇÃO}

O Handebol, prática esportiva competitiva de "organização complexa, não linear, dinâmica e auto-organizada" (Oliver, 2018, p.229), ocorre em um ambiente com elevado nível de incerteza, exigindo tomadas de decisão de alta complexidade, em função das características individuais dos jogadores, as condições da tarefa a ser resolvida e as características dos adversários (Araújo, Teques, Hernández-Mendo, Reigal, \& Anguera, 2016; Travassos, Davids, Araújo, \& Esteves, 2013). Um processo de treino efetivo para tais ações exige tarefas de treino capazes de replicar as dinâmicas e os esforços específicos com maior incidência durante o jogo (Clemente, Martins, \& Mendes, 2014; Hill-Haas, Dawson, Impellizzeri, \& Coutts, 2011), aliadas com adequadas estruturação e hierarquização de objetivos, distribuição dos conteúdos e a progressão das tarefas (Cañadas, Rodríguez, Feu, Parejo, \& García, 2013; Feu, 2006; Ibáñez, 2008; Tarodo, Belmonte, Toro, \& Ruano, 2011).

Os treinadores desempenham um papel fundamental para a efetividade no processo de treino (Ibáñez, Mendoza, \& Calvarro, 1999), tanto que estudos com treinadores esportivos tornaram-se uma das tendências emergentes na área das Ciências do Esporte (Borms, 2008), sendo observado um crescimento nas publicações em distintos países (Cushion et al., 2010; Galatti et al., 2016; Ibáñez, Pérez-Goye, García-Rubio, \& Courel-lbáñez, 2019). Além disso, o International Council for Coaches' Excellence (ICCE), organização internacional voltada ao desenvolvimento de treinadores, tem implementado ações no estabelecimento de fortes parcerias entre instituições de ensino, federações esportivas e organizações nacionais de esporte no sentido de somar esforços comuns para melhorar a formação e o desenvolvimento profissional dos treinadores em distintos níveis competitivos no mundo (ICCE, 2013).

Apesar de não existir um modelo comum de formação de treinadores no mundo, a habilitação da profissão de treinador esportivo pode ser alcançada por duas vias: a primeira baseada em cursos de educação superior nas áreas de Educação Física e/ou Ciências do Esporte e a segunda em cursos promovidos pelas federações de distintas modalidades esportivas para diferentes níveis competitivos de atuação do treinador (Falcão, Bennie, \& Bloom, 2015; Feu, 2018). Por outro lado, há uma proeminente linha investigativa indicando um consenso para o desenvolvimento profissional dos treinadores por meio de distintas formas de educação formal, não formal e informal (Jiménez, Lorenzo \& Gómez, 2009; Lyle \& Cushion, 2010; Nelson, Cushion, \& Potrac, 2006; Trudel \& Gilbert, 2006; Werthner \& Trudel, 2006; Wright, Trudel, \& Culver, 2007), evidenciando a valorização de fontes de conhecimentos procedentes das experiências da prática profissional, das experiências nas comunidades de prática e carreira esportiva, como também o acesso à literatura específica e cursos de 
formação especializada (Cunha, Estriga, \& Batista, 2014; González-Rivera, CamposIzquierdo, Villalba, \& Hall, 2017; Jiménez, Lorenzo, \& Gómez, 2009).

No Brasil, as investigações são escassas e incipientes e não apresentam uma agenda clara de pesquisa, por exemplo uma revisão sistemática realizada nos periódicos abrangendo o período de 2000 a 2015 constatou uma média de cinco artigos publicados por ano, nos quais a maior parte estava focada em aspectos relacionados a ideias dos treinadores sobre distintos assuntos e em seus comportamentos (Galatti et al., 2016). Nos estudos encontrados sobre os aspectos formativos e desenvolvimento profissional dos treinadores, os resultados enfatizam uma ampla valorização das fontes de conhecimentos procedentes dos cursos de graduação em Educação Física, com ênfase aos saberes técnicos e aos saberes advindos da Ciências do Esporte (Ramos, Graça, Nascimento, \& Silva, 2011; Rodrigues, Teixeira Costa, Santos Junior, \& Milistetd, 2017). Além disso, os estudos exclusivos com treinadores de Handebol compunham-se nas ideias dos treinadores sobre o processo de ensino-aprendizagem da modalidade em distintas categorias de formação ou na compreensão do desenvolvimento tático-técnico do jogo (Menezes, 2018; Menezes, Marques, \& Nunomura, 2017; Menezes, Ramos, Marques, \& Nunomura, 2018; Menezes, \& Reis, 2014).

As seleções brasileiras de handebol têm alcançado elevados níveis competitivos a última década, como por exemplo o título de campeão mundial da seleção feminino adulta na Sérvia em 2013 e o recente nono lugar no Campeonato Mundial masculino de 2019. Concomitante a evolução dos resultados, observou-se investimentos em centros de treinamentos, migração de atletas para as principais liga de Handebol da Europa (Asobal, Bundesliga, entre outras), além dos investimentos financeiros na preparação das seleções para os Jogos Olímpicos Rio 2016. No entanto, um fator relevante dos resultados alcançados reflete sobre a contratação de treinadores estrangeiros para dirigir as seleções adultas, como também estruturar o processo de treino das seleções nos escalões formativos (cadete, juvenil e júnior).

No caso das seleções masculinas, este processo materializou-se de forma concreta, pois o treinador estrangeiro criou acampamentos para o desenvolvimento técnico-tático de jogadores e treinadores, nos quais foi implementada uma concepção e filosofia de treino para o desenvolvimento da modalidade e para atender as demandas do modelo de jogo da seleção adulta (Amorim, 2017). Apesar de inúmeros acampamentos realizados no ciclo de preparação olímpica das seleções brasileiras (2013-2016), não foram encontrados estudos sobre o processo de formação e desenvolvimento destes treinadores, demonstrando um parco interesse da comunidade científica. No entanto, em outros países há uma preocupação constante sobre o processo de formação e desenvolvimento dos treinadores esportivos (Feu, 
2018), por exemplo sobre as fontes de conhecimentos mais significativas para os treinadores no exercício profissional e na estruturação do processo de treino do modelo de jogo das seleções masculinas (Cunha et al., 2014; Feu, Ibáñez, Calvo, Saiz, \& Cañadas, 2012; González-Rivera et al., 2017).

Diante do exposto, o estudo procurou identificar as fontes de conhecimentos (FCs) mais valorizadas pelos treinadores e treinadores assistentes no exercício profissional e na construção do processo de treino das seleções de handebol masculino nas categorias juvenil (U19), júnior (U21) e adulta.

\section{MATERIAIS E MÉTODO}

\section{Participantes}

O estudo foi classificado como estudo de caso (Montero \& León, 2007) com adesão voluntária de três treinadores e três treinadores assistentes das seleções brasileiras masculinas de Handebol das categorias juvenil (U19), júnior (U21) e adulta. Os participantes tinham em média 48,66 \$7,50 (35 - 54) anos de idade, com graduação e pós-graduação em Educação Física, exceto o treinador da seleção adulta, com formação exclusiva de treinador de handebol certificado pelo Associación de Entrenadores de Balonmano (AEBM) da Espanha. Os participantes acumulavam mais de dez anos de experiência como treinadores em níveis competitivos nacionais e internacionais. Os treinadores tinham em média 22,66 $( \pm 5,11)$ anos de experiência profissional no handebol de alto nível, enquanto os treinadores assistentes acumulavam uma média 17,66 $( \pm 6,22)$ anos, sendo que a comissão técnica da seleção U21 acumulava o menor tempo de experiência profissional no handebol de alto nível. Além disso, vale destacar que o treinador da seleção U21 e o treinador assistente da seleção U19 foram atletas profissionais da modalidade com participação em Campeonatos Mundiais e Jogos Olímpicos (Barcelona - 1992, Atlanta-1996, Atenas-2004 e Pequim - 2008).

\section{Instrumento para coleta de dados}

Para a coleta dos dados utilizou-se o Questionário de Perfil de Formação do Treinador (QPFT), originalmente proposto por Feu et al. (2012) em idioma espanhol, traduzido e adaptado para o cenário brasileiro por Mendes et al. (2019), que estabelece as procedências das fontes de conhecimento dos treinadores em três dimensões: Formação Acadêmica (AC), Experiência de Atleta (EA) e a Experiência Profissional (EP). Cada dimensão possui cinco indicadores que contemplam os momentos de aprendizagens, experiências, conhecimentos, metodologias e exercícios vivenciados pelos treinadores (Tabela 1). O instrumento é 
composto de 15 questões com respostas em escala tipo Likert de 1 a 10 (1= Discordo Plenamente e $10=$ Concordo Plenamente). Além disso, adaptou-se a pergunta original do instrumento, a qual compreende: "Para a minha atuação profissional na construção das tarefas do processo de treino das seleções, enquanto treinador eu prefiro?".

Tabela 1.

Dimensões e indicadores da versão brasileira da escala das fontes de conhecimentos dos treinadores.

\begin{tabular}{|c|c|}
\hline Dimensões & $\begin{array}{l}\text { Para a minha atuação profissional e na construção das tarefas do processo de } \\
\text { treino das seleções, enquanto treinador eu prefiro?" }\end{array}$ \\
\hline $\begin{array}{l}\text { Experiência } \\
\text { Atleta }\end{array}$ & $\begin{array}{l}\text { I) As experiências que adquiri ao longo da minha carreira como atleta. } \\
\text { II) Os conhecimentos adquiridos com os treinadores que me treinaram. } \\
\text { III) Métodos que eram utilizados pelos meus treinadores. } \\
\text { IV) O que aprendi com outros treinadores enquanto era atleta. } \\
\text { V) Os exercícios e tarefas que aprendi quando era atleta. }\end{array}$ \\
\hline $\begin{array}{l}\text { Experiência } \\
\text { Profissional }\end{array}$ & $\begin{array}{l}\text { I) A metodologia que criei a partir da minha própria experiência à frente do } \\
\text { processo de treino. } \\
\text { II) A experiência adquirida com os exercícios criados por mim no processo de } \\
\text { treino. } \\
\text { III) Pôr em prática as teorias elaboradas com base na minha experiência pessoal } \\
\text { como treinador. } \\
\text { IV) Os conhecimentos adquiridos ao longo do meu trabalho e da minha } \\
\text { experiência como treinador. } \\
\text { V) O que aprendi por iniciativa própria sem auxílio de cursos ou de outras } \\
\text { pessoas. }\end{array}$ \\
\hline $\begin{array}{l}\text { Formação } \\
\text { Acadêmica }\end{array}$ & $\begin{array}{l}\text { I) Os critérios metodológicos aprendidos em cursos de formação de treinadores. } \\
\text { II) O que aprendi durante a minha formação acadêmica na área de Educação } \\
\text { Física. } \\
\text { III) Os conhecimentos aprendidos nos cursos de formação que participei. } \\
\text { IV) Os exercícios e tarefas aprendidos durante os cursos de formação de } \\
\text { treinadores. } \\
\text { V) O que aprendi durante a minha formação como treinador. }\end{array}$ \\
\hline
\end{tabular}

Fonte: Elaborado pelo autores, 2020. 


\section{Procedimentos para coleta de dados}

Para a aplicação da versão brasileira do QPFT, o estudo foi submetido e aprovado (Parecer $\mathrm{n}^{0}$ 1835025) pelo Comitê de Ética em Pesquisa com Seres Humanos da Universidade Estadual do Oeste do Paraná (UNIOESTE). Os treinadores e treinadores assistentes foram convidados durante as etapas de treino de suas respectivas seleções nas temporadas esportiva de 2016 e 2017. Na sequência, os questionários foram enviados por meio de correio eletrônico com a opção de preenchimento do documento em formato Word do Office 2003 ou via formulário do Google Docs.

\section{Análise de dados}

A distribuição dos dados apresentou valores discrepantes, caracterizando uma amostra não normal dos resultados. Assim, optou-se por uma análise individualizada dos dados, considerando o nível de valorização indicado pelos treinadores e treinadores assistentes em cada item do instrumento, com posterior cálculo da mediana geral dos indicadores de acordo com os resultados dos treinadores e treinador assistentes de forma separada. Os valores de mediana maiores ou iguais a oito $(8,0)$ foram adotados para considerar os itens mais valorizados pelos treinadores e treinadores assistentes em relação a procedência das fontes de conhecimento. Apesar do número reduzido de treinadores no estudo, procurou-se identificar diferenças na valorização das FCs entre os treinadores e os treinadores assistentes por meio da prova $U$ de Mann Whitney e na comparação dos resultados entre as comissões técnicas (treinador e treinador assistente) de cada seleção, por meio do e o teste de Kruskal Wallis (Leotti, Coster, \& Riboldi, 2012). Os dados foram analisados com o auxílio do software SPSS 23 (Statistics for Windows, Version 23.0) e as figuras construídas nas planilhas eletrônicas de cálculos no software Office 2016.

\section{RESULTADOS}

Os itens relacionados com as fontes de conhecimentos (FCs) procedentes da Experiência Profissional (EP) e da Formação Acadêmica ( $F A$ ) foram mais valorizadas entre os treinadores das seleções (Figura 1). 


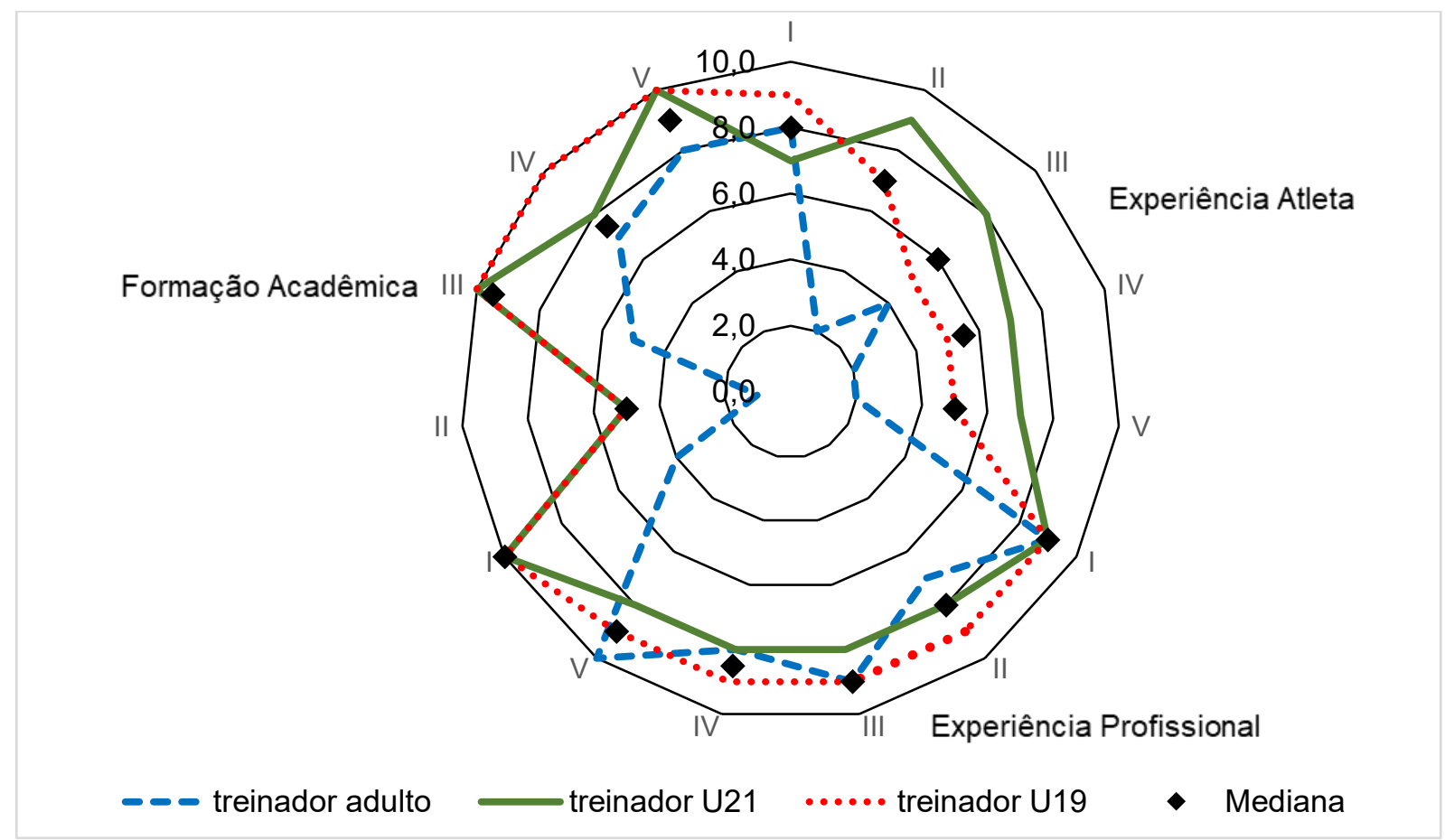

Figura 1. Mediana e indicadores de valorização em relação a procedência das fontes de conhecimento dos treinadores das seleções U19, U21 e adulta. Nota: I, II, III, IV e V são os itens das três dimensões do instrumento. Fonte: Elaborado pelo autores, 2020.

Entretanto, o treinador da seleção adulta valoriza mais as FCs procedentes da EP, em especial aos itens relacionados com os aspectos metodológicos e conhecimentos teóricos adquiridos por inciativa própria e edificados das experiências profissionais, enquanto os treinadores das seleções U19 e U21, além das FCs procedentes da EP, também valorizavam as FCs procedentes da FA, como os itens relacionados aos conhecimentos e aos aspectos metodológicos adquiridos em cursos de formação inicial em Educação Física e de formação e capacitação de treinadores.

O mesmo fato ocorreu nos resultados dos treinadores assistentes (Figura 2), com maior valorização dos itens relacionados as fontes de conhecimentos (FCs) procedentes da Experiência Profissional (EP) e da Formação Acadêmica (FA). Em especial, aos itens relacionados aos aspectos metodológicos e conhecimentos teóricos adquiridos por inciativa própria a partir das experiências profissionais e aos itens relacionados aos conhecimentos e aspectos metodológicos adquiridos em cursos de formação e capacitação de treinadores. 


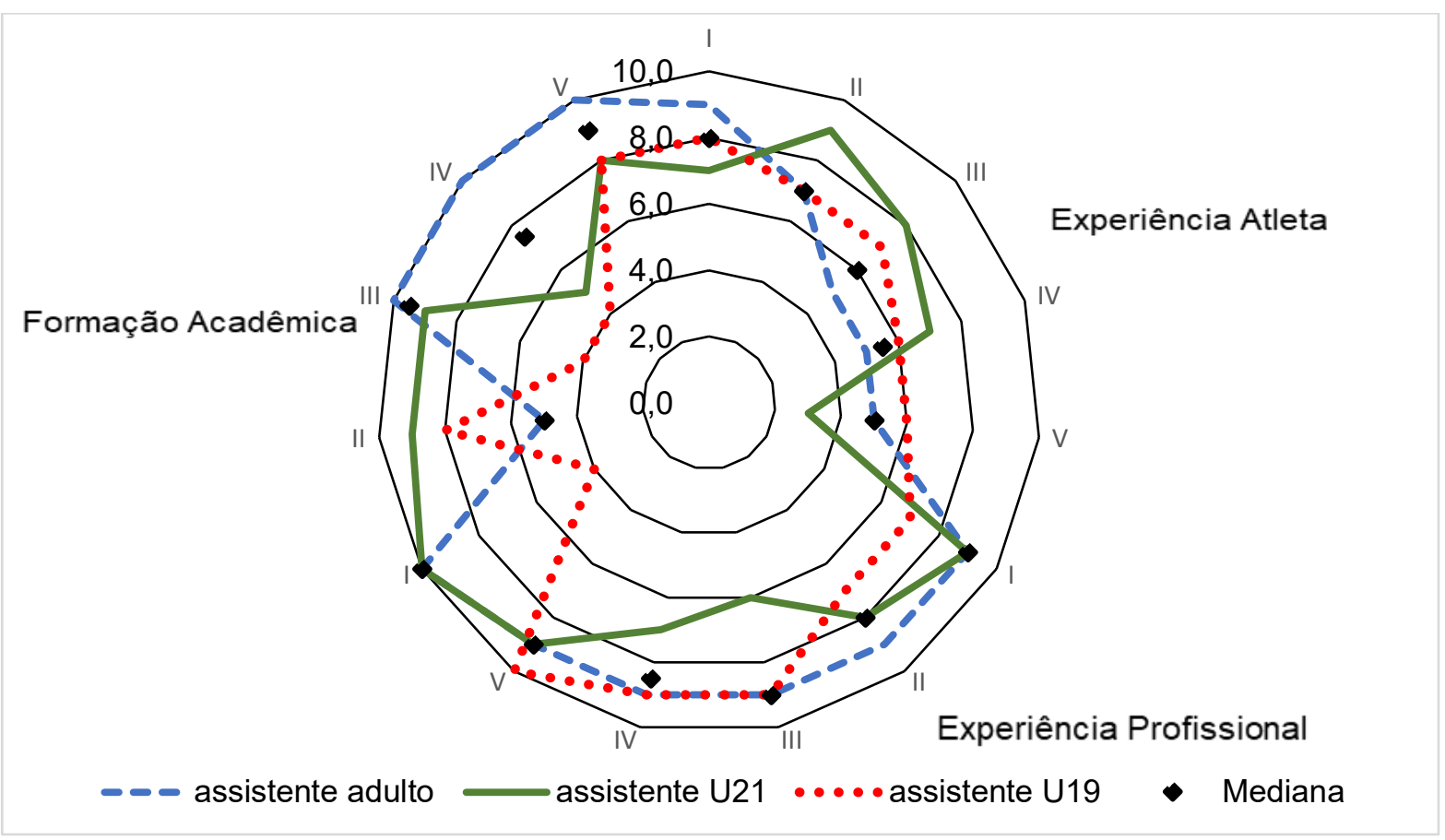

Figura 2. Mediana e indicadores de valorização da procedência das fontes de conhecimento dos treinadores assistentes das seleções U19, U21 e adulta. Nota: I, II, III, IV e V são os itens das três dimensões do instrumento. Fonte: Elaborado pelo autores, 2020.

Por outro lado, quando analisados os resultados das comissões técnicas (treinador e treinador assistente) de cada seleção (igura 3), observou-se que os itens relacionados as FCs procedentes da EP foram mais valorizados pelos integrantes das comissões técnicas das seleções U19 (Me=9,0) e adulta ( $M e=9,0)$, enquanto os membros da comissão técnica da seleção U21 (Me=9,5) valorizavam mais os itens relacionados as FCs procedentes da FA.

No entanto, não foram encontradas diferenças significativas nos resultados do teste de Kruskal Wallis entre as FCs, independentemente serem procedentes da EP $\left(X^{2}=5,00\right.$; $p>0,05)$, FA $\left(X^{2}=0,32 ; p>0,05\right)$ ou da EA $\left(X^{2}=3,58 ; p>0,05\right)$ entre as comissões técnicas das seleções U19, U21 e adulta. 


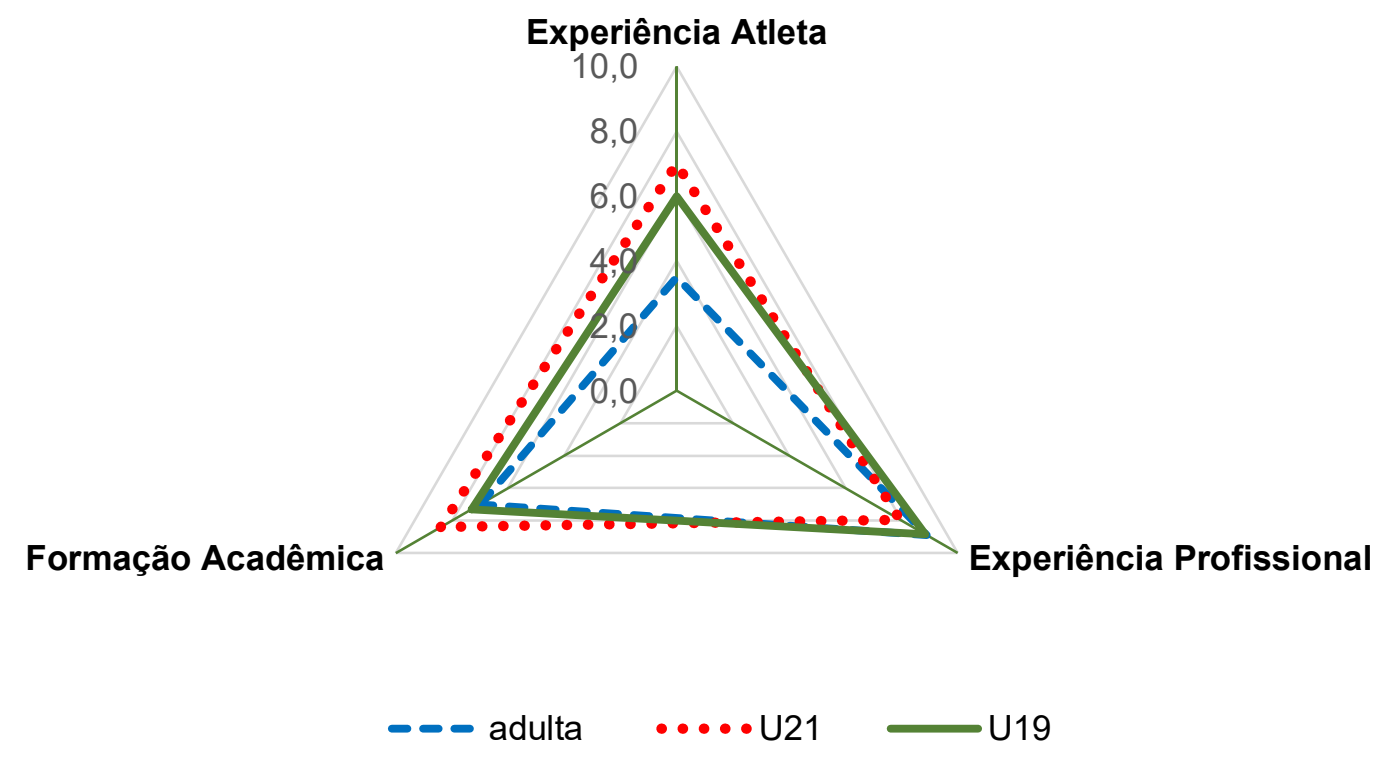

Figura 3. Mediana da valorização das FCs das comissões técnica das seleções U19, U21 e adulta. Fonte: Elaborado pelos autores, 2020.

Dentre outros resultados relevantes identificados, vale ressaltar a baixa valorização dos itens relacionados as fontes de conhecimentos procedentes da Experiência de Atleta pelos treinadores e treinadores assistentes das seleções U19, U21 e adulta, como também a ausência de diferenças significativas nos resultados na prova $U$ de Mann Whitney na valorização dos itens das $F C$ s procedentes da $\operatorname{EP}(U=4,50 ; p>0,05)$, $F A(U=3,50 ; p>0,05)$ e EA $(U=2,50 ; p>0,05)$, quando comparados os resultados entre os treinadores e os treinadores assistentes das seleções U19, U21 e adulta.

\section{DISCUSSÃO}

A partir do objetivo de identificar as fontes de conhecimentos mais valorizadas pelos treinadores e treinadores assistentes no exercício profissional e na construção do processo de treino das seleções de handebol masculino nas categorias U19, U21 e adulta, os resultados encontrados ratificam as ideias de alguns autores (Cunha et al., 2014; Cushion, Armour, \& Jones, 2003), que as pesquisas com treinadores adquirem características específicas do contexto regional, sem desprezar as vertentes individuais e sociais inerente ao processo de treino esportivo. No caso do Brasil, os aspectos relacionados as exigências legais de formação inicial em Educação Física para o exercício legal da profissão de treinador, têm 
despertado o interesse e a curiosidade da comunidade científica internacional sobre a realidade brasileira (Milistetd, Trudel, Mesquita, \& Nascimento, 2014).

No caso das seleções brasileiras masculinas de handebol, constatou-se uma valorização similar entre as FCs procedentes da EP e FA e valorização superior destas em relação as FCs procedentes da EA. Apesar do estudo confirmar os resultados de investigações internacionais, evidenciando a valorização de fontes de conhecimentos procedentes das experiências da prática profissional (Cushion et al., 2003; Irwin, Hanton, \& Kerwin, 2004; Stoszkowski \& Collins, 2016; Wright et al., 2007), ainda permanece a alta valorização das FCs procedentes da "formação acadêmica" (aprendizagem formal) pelos treinadores e treinadores assistentes para a atuação profissional e na construção do processo de treino do modelo de jogo das seleções masculinas de Handebol.

As exigências legais de realização do curso de graduação em Educação Física, enquanto requisito obrigatório para a atuação profissional na maioria das modalidades esportivas no Brasil, exceto para treinadores de futebol profissional e de algumas artes marciais/lutas, têm suscitado uma expectativa de que uma formação em nível superior seja traduzida em melhor qualificação e maior reconhecimento social para a profissão de treinador (ICCE, 2013). No entanto, estudos tem apontado um baixo impacto dos cursos de graduação na formação de treinadores esportivos no Brasil (Ramos et al., 2011; Rodrigues et al., 2017), embora exista um robusto corpo de conhecimentos profissionais desenvolvidos nestes cursos, a formação universitária parece limitar-se a preparar treinadores para atuar somente no contexto de participação esportiva e proporciona poucas oportunidades diversificada de experienciais de aprendizagem (Milistetd et al., 2014).

Além disso, a comunidade científica internacional tem feito um apelo veemente para adesão a programas de desenvolvimento profissional de treinadores, em especial aqueles que integrem situações de aprendizagem mediadas, não-mediadas e internas (Werthner \& Trudel, 2006), que estimulem uma prática reflexiva dos treinadores (Gilbert \& Trudel, 2001; Leggett \& James, 2016; Nash \& Sproule, 2012), capacitando-os para realizar as distintas adaptações no complexo contexto de um processo de treinamento.

Por outro lado, as FCs procedentes da EA foram as menos valorizadas entre os treinadores e treinadores assistentes investigados, mesmo com as elevadas experiências atléticas competitivas internacionais vivenciadas pelo treinador da seleção U21 e o treinador assistente da seleção U19 em Jogos Olímpicos e Campeonatos Mundiais. Neste caso, os resultados podem indicar que estas experiências podem estar aliadas a outras situações distintas do processo de treino, como em tomadas de decisão nas relações interpessoais treinador/treinador assistente/atleta (Côté \& Gilbert, 2009). 
Apesar da experiência atlética não ser uma condição necessária para a formação de treinadores de elite, há o reconhecimento de que ela contém elementos importantes na aquisição de conhecimentos para o exercício profissional (Mesquita, Isidro, \& Rosado, 2010; Mesquita, Jones, Fonseca, \& Silva, 2012). Portanto, seria fundamental o incentivo a vivências de situações distintas de aprendizagem em contextos informais em programas de formação e desenvolvimentos dos treinadores brasileiros, como por exemplo, a valorização de experiência como atleta, a experiência prática profissional, a participação em cursos de treinadores, a supervisão de mentores e a interação com outros treinadores (Cushion et al., 2003; Irwin et al., 2004; Stoszkowski \& Collins, 2016; Wright et al., 2007).

Embora não tenham sido observadas diferenças significativas na comparação das FCs de treinadores e treinadores assistentes, observou-se uma maior valorização das FCs procedentes da EP, ou seja, por meio de situações de aprendizagem informal. A permanência do treinador de nacionalidade espanhola, com formação distinta dos treinadores brasileiros, na direção da seleção adulta masculina por quase uma década e visando a participação nos Jogos Olímpicos de Pequim 2008 e Rio 2016, parece ter afetado os demais treinadores investigados. Neste caso, a qualificação e o reconhecimento internacional do treinador da seleção adulta podem ter facilitado o acesso dos demais integrantes das comissões técnicas das seleções às experiências de aprendizagem em contextos informais.

As experiências de aprendizagem em contexto informais, como por exemplo oportunidades para a interação e observação de treinadores de elite da modalidade, são reconhecidas como fontes do conhecimento de elevada contribuição para o desenvolvimento de treinadores de elite mundial (Erickson, Bruner, MacDonald, \& Côté, 2008; Irwin et al., 2004; Rynne, 2012). Assim, a dinamicidade e o elevado nível das exigências competitivas internacionais, aliadas a experiência acumulada na atuação profissional do handebol de elite, podem ser consideradas contínuas oportunidades de aprendizagem para os treinadores e treinadores assistentes das seleções brasileiras (Cushion et al., 2010; Hanratty \& O'Connor, 2012; Occhino, Mallett, \& Rynne, 2013; Rynne, Mallett, \& Tinning, 2010).

Investigações similares com treinadores de distintas modalidades no Brasil também revelaram a maior valorização das FCs procedentes da EP, com ênfase nos meios da aprendizagem informal (Ramos et al., 2011; Rodrigues et al., 2017), o que parece consenso em investigações internacionais com treinadores de Handebol (Cunha et al., 2014; Feu et al., 2012) e outras modalidades (González-Rivera et al., 2017; Mesquita et al., 2010).

Os resultados encontrados compreendem importantes subsídios a serem compartilhados com a Confederação Brasileira de Handebol, no sentido de favorecer a implementação de estratégias de formação a longo prazo dos treinadores, em especial 
aquelas que permitam mudanças nas estruturas cognitivas dos treinadores e fomentem a reflexão sobre a sua prática profissional. Apesar das limitações do estudo, principalmente a utilização somente de dados dos treinadores das seleções masculinas e nas temporadas esportivas de 2016 e 2017, as evidências refletem a natureza complexa da atuação profissional dos treinadores de maior expressão da modalidade no país, podendo ser resultantes do desenvolvimento profissional de treinadores esportivos no Brasil.

\section{CONCLUSÕES}

A atuação profissional dos treinadores e treinadores assistentes das seleções brasileiras masculinas das categorias U19, U21 e adulta de Handebol e a construção do processo de treino do modelo de jogo das seleções masculinas de Handebol estruturam-se a partir das fontes de conhecimentos procedentes da "experiência profissional". Entretanto, ainda há elevada valorização das fontes de conhecimentos procedentes da "formação acadêmica", consideradas fontes de conhecimentos com menor influência no processo de formação e desenvolvimento dos treinadores de elite internacionais.

A alta valorização do contexto formal de aprendizagem pelos treinadores investigados, em especial pelos integrantes da comissão técnica da seleção U21, pode estar relacionada ao sancionamento da Lei N. 9696/98, que limita a atuação profissional de treinadores esportivos após a obtenção do grau acadêmico de Bacharel em Educação Física, como também as poucas oportunidades de situações de aprendizagem que superem as perspectivas tradicionais de ensino adotadas nos programas de desenvolvimento de treinadores.

Os resultados encontrados indicam a necessidade da expansão de investigações envolvendo treinadores de Handebol do Brasil em distintos níveis competitivos, como também em outras modalidades esportivas, em especial os estudos que possam servir de suporte para a atuação dos treinadores em sua realidade prática. Além disso, o processo de formação e desenvolvimento de treinadores a longo prazo deveria ser uma preocupação imediata e constante das entidades administrativa do esporte no país. 


\section{REFERÊNCIAS}

Amorim, A.M. (2017). Desenvolvimento tático-técnico no handebol masculino: estratégias utilizadas nos acampamentos nacionais promovidos pela Confederação Brasileira de Handebol (Tese de Doutoramento). Universidade Federal de Santa Catarina. Recuperado de https://repositorio.ufsc.br/handle/123456789/189453

Araújo, D., Teques, P., Hernández-Mendo, A., Reigal, R.E., \& Anguera, M.T. (2016). La toma de decisión, ¿es una conducta observable?: Discusión sobre diferentes perspectivas teóricas utilizadas en el estudio del rendimiento deportivo. Cuadernos de Psicología del Deporte, 16(1), 183-196. Recuperado de https://revistas.um.es/cpd/article/view/254481

Borms, J. (2008). Directory of sport science: A journey through time: the changing face of ICSSPE (5 $5^{\mathrm{a}}$ ed.). Ilinois, Estados Unidos: Human Kinetics.

Cañadas, M., Rodríguez, G., Feu, S., Parejo, I., \& García, J. (2013). Relathionship between pedagogical content knowledge and coaching methods. Revista de psicologia del deporte, 22(1), 183-186. Recuperado de https://www.redalyc.org/pdf/2351/235127552017.pdf

Clemente, F., Martins, F.M., \& Mendes, R.S. (2014). Periodization based on small-sided soccer games: Theoretical considerations. Strength \& Conditioning Journal, 36(5), 3443. doi: https://doi.org/10.1519/SSC.0000000000000067

Côté, J., \& Gilbert, W. (2009). An integrative definition of coaching effectiveness and expertise. International Journal of Sports Science \& Coaching, 4(3), 307-323. doi: https://doi.org/10.1260\%2F174795409789623892

Cunha, A.F.V.P., Estriga, M.L.D., \& Batista, P.M.F. (2014). Fontes de conhecimento percebidas pelos treinadores: estudo com treinadores de andebol da $1^{\text {a }}$ divisão de seniores masculinos em Portugal. Movimento: Revista de Educação Física da UFRGS, 20(3), 917-940. doi: https://doi.org/10.22456/1982-8918.43663

Cushion, C., Armour, K., \& Jones, R. (2003). Coach education and continuing professional development: Experience and learning to coach. Quest, 55(3), 215-230. doi: https://doi.org/10.1080/00336297.2003.10491800

Cushion, C., Nelson, L., Armour, K., Lyle, J., Jones, R., Sandford, R., \& O'Callaghan, C. (2010). Coach learning and development: $A$ review of literature. London: The national Coaching

Foundation.

Recuperado

de 
https://www.researchgate.net/publication/265566741 Coach Learning and Developm ent A Review of Literature

Erickson, K., Bruner, M.W., MacDonald, D.J., \& Côté, J. (2008). Gaining insight into actual and preferred sources of coaching knowledge. International Journal of Sports Science \& Coaching, 3(4), 527-538. doi: https://doi.org/10.1260\%2F174795408787186468

Falcão, W.R., Bennie, A., \& Bloom, G A. (2015). Desporto de alto rendimento: formação e competências do treinador. Em: Formação e Saberes em Desporto, Educação Física

Feu, S. (2006). Organización didáctica del proceso de enseñanza-aprendizaje para la construcción del juego ofensivo en balonmano. E-Balonmano.com: Revista de Ciencias del Deporte, 2(4). Recuperado de http://www.ebalonmano.com/ojs/index.php/revista/article/view/12/10

Feu, S. (2018). El aprendizage del balonmano en la edad escolar desde el Modelo Tactical Game. Em: S. Feu, J. García-Rubio, \& S.J.G. Ibañez (Eds.), Avances cientificos para el aprendizage y desarrolo del Balonmano. Caceres, Brasil: Editora da UNEMAT.

Feu, S., Ibáñez, S.J., Calvo, A.L., Saiz, S.J., \& Cañadas, M. (2012). El conocimiento profesional adquirido por el entrenador de balonmano: experiencias y formación. Revista de psicologia del deporte, 21(1), 107-115. Recuperado de https://bit.ly/3jugTiZ

Galatti, L., Bettega, O.B., Brasil, V.Z., de Souza Sobrinho, A.E.P., Bertram, R., Tozetto, A.V.B., ... Milistetd, M. (2016). Coaching in Brazil Sport Coaching as a Profession in Brazil: An Analysis of the Coaching Literature in Brazil From 2000-2015. International Sport Coaching Journal, 3(3), 316-331. doi: https://doi.org/10.1123/iscj.2015-0071

Gilbert, W.D., \& Trudel, P. (2001). Learning to coach through experience: Reflection in model youth sport coaches. Journal of teaching in physical education, 21(1), 16-34. Recuperado de https://bit.ly/3kMw48Z

González-Rivera, M.D., Campos-Izquierdo, A., Villalba, A.I., \& Hall, N.D. (2017). Sources of knowledge used by Spanish coaches: A study according to competition level, gender and professional experience. International Journal of Sports Science \& Coaching, 12(2), 162-174. doi: https://doi.org/10.1177\%2F1747954117694733

Hanratty, M., \& O'Connor, D. (2012). Understanding Expert Knowledge: A Case Study of Knowledge Acquisition in Elite Rugby League Strength and Conditioning Coaches. International Journal of Coaching Science, 6(1), 45-63. 
Hill-Haas, S.V., Dawson, B., Impellizzeri, F.M., \& Coutts, A.J. (2011). Physiology of small-sided games training in football: a systematic review. Sports Medicine, 41(3), 199-220. doi: https://doi.org/10.2165/11539740-000000000-00000

Ibáñez, S.J. (2008). La planificación y el control del entrenamiento técnico-táctico en baloncesto. En: N. Terrados \& J. Calleja (Eds.), Fisiología, entrenamiento y medicina del baloncesto (pp. 299-313). Barcelona: Paidotribo.

Ibáñez, S.J., Mendoza, M.Á.P., \& Calvarro, J.M.A. (1999). Taxonomía de medios para la iniciación al baloncesto. Red: revista de entrenamiento deportivo, 13(4), 15-24.

Ibáñez, S.J., Pérez-Goye, E., García-Rubio, J., \& Courel-lbáñez, J. (2019). Effects of task constraints on training workload in elite women's soccer. International Journal of Sports Science \& Coaching, 15(1), 90-107. doi: https://doi.org/10.1177/1747954119891158

International Council for Coaching Excellence (ICCE). (2013). International Sport Coaching Framework Version 1.2. Champaign, United States: Human Kinetics. Recuperado de https://www.icce.ws/ assets/files/iscf-1.2-10-7-15.pdf

Irwin, G., Hanton, S., \& Kerwin, D. (2004). Reflective practice and the origins of elite coaching knowledge. Reflective Practice, 5(3), 425-442. doi: https://doi.org/10.1080/1462394042000270718

Jiménez, S., Lorenzo, A., \& Gómez , M. Á. (2009). Medios de formación de los entrenadores expertos en baloncesto. Cultura Ciencia Deporte [CCD], 4(11), 119-126. Recuperado de https://dialnet.unirioja.es/servlet/articulo?codigo=3097154

Leggett, R., \& James, J. (2016). Exploring the Benefits of a Coach Development Process ... on the Coach. International Journal of Human Resource Development: Practice, Policy \& Research, 1(2), 55-65. doi: https://doi.org/10.22324/ijhrdppr.1.116

Leotti, V. B., Coster, R., \& Riboldi, J. (2012). Normalidade de variáveis: métodos de verificação e comparação de alguns testes não-paramétricos por simulação. Revista HCPA, 32(2), 227-234. Recuperado de https://seer.ufrgs.br/hcpa/article/view/29874/19186

Lyle, J., \& Cushion, C. (Eds.). (2010). Sports Coaching: Professionalisation and Practice is a comprehensive evidence-based textbook of sports coaching theory and practice.

Mendes, J. C., Brandt, R., Carvalho, H. M., Feu, S., Ibañez, S. J., \& Nascimento, J. V. (2019). Validation and reliability of a sources of knowledge scale for Brazilian coaches. Motriz: 
Revista de Educação Física, 25(3). doi: http://dx.doi.org/10.1590/S1980$\underline{6574201900030013}$

Menezes, R. P. (2018). Ensino do handebol em longo prazo: estudo a partir da opinião de treinadores. Educación Física y Ciencia, 20(2). Recuperado de http://sedici.unlp.edu.ar/handle/10915/70622

Menezes, R. P., Marques, R. F. R., \& Nunomura, M. (2017). Teaching handball to players under-12: the perspective of Brazilian coaches. Motriz: Revista de Educação Física, 23(4), 1-8. doi: https://doi.org/10.1590/s1980-6574201700040006

Menezes, R. P., Ramos, N. C., Marques, R. F. R., \& Nunomura, M. (2018). Teaching handball to $\mathrm{U}-16$ and $\mathrm{U}-18$ women's teams: coaches' perspective on the long-term. Motriz: Revista de Educação Física, 24(4). doi: http://dx.doi.org/10.1590/S1980$\underline{6574201800040001}$

Menezes, R. P., \& Reis, H. H. B. (2014). Relação entre eficácia defensiva e elementos técnicotáticos do handebol a partir dos discursos de técnicos experientes. Journal of Physical Education, 25(4), 513-526. doi: https://doi.org/10.4025/reveducfis.v25i4.21709

Mesquita, I., Isidro, S., \& Rosado, A. (2010). Portuguese coaches' perceptions of and preferences for knowledge sources related to their professional background. Journal of Sports Science \& Medicine, 9(3), 480-489. Recuperado de https://www.ncbi.nlm.nih.gov/pmc/articles/PMC3761720/

Mesquita, I., Jones, R., Fonseca, J., \& Silva, L. D. M. (2012). Nova abordagem na formação de treinadores; O que mudou e porquê?. Em In construção da identidade profissional em educação física. A formação á intervenção (pp. 41-60).

Milistetd, M., Trudel, P., Mesquita, I., \& Nascimento, J. V. (2014). Coaching and coach education in Brazil. International Sport Coaching Journal, 1(3), 165-172.

Montero, I., \& León, O. G. (2007). A guide for naming research studies in Psychology. International Journal of Clinical and Health Psychology, 7(3), 847-862. Recuperado de https://www.redalyc.org/pdf/337/33770318.pdf

Nash, C., \& Sproule, J. (2012). Coaches perceptions of their coach education experiences. International Journal of Sport Psychology, 43(1), 33-52. Recuperado de https://www.researchgate.net/publication/234092162 Coaches perceptions of their c oach education experiences 
Nelson, L. J., Cushion, C. J., \& Potrac, P. (2006). Formal, nonformal and informal coach learning: A holistic conceptualisation. International Journal of Sports Science \& Coaching, 1(3), 247-259. doi: https://doi.org/10.1260\%2F174795406778604627

Occhino, J., Mallett, C., \& Rynne, S. (2013). Dynamic social networks in high performance football coaching. Physical Education and Sport Pedagogy, 18(1), 90-102. doi: https://doi.org/10.1080/17408989.2011.631003

Oliver, J. F. C. (2018). El balonmano deporte de organización compleja. El error un componente de la auto organización del juego: una propuesta de entrenamiento. Em S. Feu, J. García \& S. J. Ibáñez (Coords.), Avances científicos para el aprendizaje y desarrollo del balonmano. Caceres, España: Universidad de Extremadura.

Ramos, V., Graça, A., Nascimento, J. V., \& Silva, R. (2011). A aprendizagem profissional-as representações de treinadores desportivos de jovens: quatro estudos de caso. Motriz: Revista de Educação Física, 17(2), 280-291. doi: http://dx.doi.org/10.5016/1980$\underline{6574.2011 \mathrm{v} 17 \mathrm{n} 2 \mathrm{p} 280}$

Rodrigues, H. A., Teixeira Costa, G.C., Dos Santos Junior, E. L., \& Milistetd, M. (2017). As fontes de conhecimento dos treinadores de jovens atletas de basquetebol. Motrivivência, 29(51). doi: https://doi.org/10.5007/2175-8042.2017v29n51p100

Rynne, S. B. (2012). Performance coaching craft through work and study. Em P. Potrac, W. Gilbert \& J. Denison (Eds.), Routledge handbook of sports coaching. Londres: Routledge.

Rynne, S. B., Mallett, C. J., \& Tinning, R. (2010). Workplace learning of high performance sports coaches. Sport, Education and Society, 15(3), 315-330. doi: https://doi.org/10.1080/13573322.2010.493312

Stoszkowski, J., \& Collins, D. (2016). Sources, topics and use of knowledge by coaches. Journal of Sports Sciences, 34(9), 794-802. doi: https://doi.org/10.1080/02640414.2015.1072279

Tarodo, J., Belmonte, M. J., Toro, E., \& Ruano, M. Á. (2011). Opinión de los entrenadores sobre distribución de contenidos técnico-tácticos y pedagógicos en distintas categorías de baloncesto de formación. Cuadernos de Psicología del Deporte, 11(2), 51-62. Recuperado de https://www.redalyc.org/articulo.oa? id=227019296005 
Travassos, B., Davids, K., Araújo, D., \& Esteves, T. P. (2013). Performance analysis in team sports: Advances from an Ecological Dynamics approach. International Journal of Performance Analysis in Sport, 13(1), 83-95. doi: https://doi.org/10.1080/24748668.2013.11868633

Trudel, P., \& Gilbert, W. (2006). Coaching and coach education. Handbook of physical

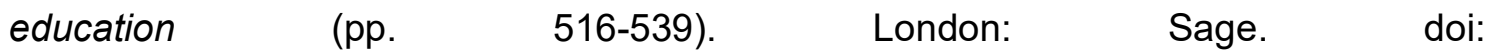
https://doi.org/10.4135/9781848608009.n29

Werthner, P., \& Trudel, P. (2006). A new theoretical perspective for understanding how coaches learn to coach. The Sport Psychologist, 20(2), 198-212. Recuperado de https://www.researchgate.net/publication/267936822 A New Theoretical Perspective for Understanding How Coaches Learn to Coach

Wright, T., Trudel, P., \& Culver, D. (2007). Learning how to coach: the different learning situations reported by youth ice hockey coaches. Physical Education and Sport Pedagogy, 12(2), 127-144. doi: https://doi.org/10.1080/17408980701282019 